\title{
Prevalencia de osteoporosis en ancianos institucionalizados, mediante ultrasonografía de calcáneo
}

\author{
F. J. NAVAS CÁMARA, F. J. FERNÁNDEZ DE SANTIAGO ${ }^{1,4}$, I. BAYONA MARZO², \\ T. MINGO GÓMEZ ${ }^{3}$, M. M. DE LA FUENTE SANZ ${ }^{3}$ A. CACHO DEL AMO ${ }^{4}$
}

Dpto. Bioquímica, Biología Molecular y Fisiología. 'Dpto. Anatomía y Radiología. ${ }^{2} D$ pto. Anatomía Patológica, Microbiología, Medicina Preventiva y Salud Pública, Medicina Legal y Forense. ${ }^{3}$ Dpto. Cirugía, Oftalmología, Otorrinolaringología y Fisioterapia. E.U. Fisioterapia de Soria. Universidad de Valladolid. ${ }^{4}$ Residencia de la Tercera Edad "El Parque”. Soria

\begin{abstract}
PREVALENCE OF OSTEOPOROSIS ASSESSED BY OUANTITATIVE ULTRASOUND CALCANEUS MEASUREMENTS IN INSTITUTIONALIZED ELDERLY POPULATION
\end{abstract}

\section{RESUMEN}

Objetivos: Analizar mediante ultrasonografía cuantitativa de calcáneo (QUS) la prevalencia de osteoporosis en ancianos institucionalizados, para contribuir al establecimiento de un punto de corte en unidades de T-score en el diagnóstico de osteoporosis, lo que permitiría avanzar en la prevención de fracturas.

Material y métodos: Se analizó mediante QUS a la población $(\mathrm{n}=$ 171) por sexos y por grupos de edad (tres décadas entre los 70 y los 90 años).

Resultados: Para todas las variables densitométricas, tanto de forma global como en los grupos de edad más jóvenes, las mujeres mostraron valores significativamente más bajos que los varones $(\mathrm{p}<0,001)$. En el grupo de mayor edad sólo se aprecian diferencias significativas en la Tscore y en el BUA ( $p=0,039$ y $p=0,025$, respectivamente). Bajo cualquiera de los criterios aplicados y en todos los grupos de edad, la prevalencia en las mujeres es superior a la del varón. Aplicando los criterios de la OMS con QUS, la prevalencia de osteoporosis en la población total se acerca a la establecida mediante DEXA con esos mismos criterios.

Conclusión: La técnica QUS puede ser muy útil para valorar la evolución de la masa ósea con la edad y para el diagnóstico y seguimiento de la osteoporosis. En nuestra población anciana, los criterios de la OMS para la técnica DXA, son también los más adecuados para el empleo de la técnica QUS.

PALABRAS CLAVE: Osteoporosis. Ultrasonografía. Calcáneo. Anciano.

Navas Cámara FJ, Fernández de Santiago FJ, Bayona Marzo I, Mingo Gómez T, de la Fuente Sanz MM, Cacho del Amo A. Prevalencia de osteoporosis en ancianos institucionalizados, mediante ultrasonografía de calcáneo. An Med Interna (Madrid) 2006; 23: 374-378.
ABSTRACT

Objectives: To analyze using quantitative ultrasound of calcaneus (QUS) the prevalence of osteoporosis in institutionalized elderly people, in order to contribute to state reliable criteria (T-score units) for the diagnosis of osteoporosis which allow advances in bone fracture prevention.

Material and methods: Elderly people $(n=171)$ were divided into separate groups according to sex and age criteria (three decades, from 70 to 90 years) and analyzed using QUS.

Results: Both globally and in the younger groups, women showed significantly lower values than men in all densitometry variables $(p<$ $0.001)$. In the oldest group only $T$-score and BUA showed statistical differences ( $p=0.039$ y $p=0.025$, respectively). The prevalence of osteoporosis in women was higher than in men whichever criteria were applied in all age groups. Applying the WHO criteria with QUS, the prevalence of osteoporosis in global population is close to that stated by DEXA using the same criteria.

Conclusions: QUS could be useful to assess the bone mass evolution with age and for the diagnosis and monitoring of osteoporosis. In our elderly population, the WHO criteria for DEXA, are also the most suitable ones for QUS utilization.

KEY WORDS: Osteoporosis. Ultrasound. Calcaneus. Elderly people.

\section{INTRODUCCIÓN}

La osteoporosis se ha denominado "la gran epidemia silenciosa del siglo XX". Su prevalencia ha aumentado en los últimos años y continuará haciéndolo de forma exponencial, acompañando al progresivo envejecimiento de la población. Es una enfermedad frecuentemente asintomática, que suele debutar con su peor complicación, la fractura, como conse- cuencia de un incremento de la fragilidad ósea debida a la progresiva desmineralización del hueso y al cambio de su microarquitectura (1-3).

En España, esta enfermedad la padecen alrededor de 2,5 millones de mujeres de las que un $40 \%$ no están diagnosticadas, encontrándose por tanto fuera del control sanitario. Por otra parte, la Asociación Española Contra la Osteoporosis (AECOS) informa que la incidencia anual de fracturas de

Trabajo aceptado: 27 de marzo de 2006 
cadera en nuestro país es de 60.000, y ello supone una sangría económica importante para la sanidad pública. En la Unión Europea se espera que la incidencia anual de fractura de cadera pase de 414.000 a 972.000 casos en los próximos 50 años (4). La puesta en marcha de medidas preventivas y el establecimiento de un diagnóstico y un tratamiento precoz podrían disminuir estas cifras de forma significativa.

La ultrasonografía cuantitativa de calcáneo (QUS), se puso en marcha hace ya dos décadas y es una técnica barata, rápida, practicable con equipos portátiles que no irradian ni precisan personal especializado, y que junto a los datos de la historia clínica, nos acercan al estado del hueso del paciente (5). Aunque la QUS se trata de una prueba propuesta como alternativa a la densitometría ósea por absorciometría dual de rayos X (DXA) (considerada ésta como la "prueba de oro" en el diagnóstico de osteoporosis) (6), es una técnica que no ha terminado de instaurarse en la práctica clínica.

Por otra parte, en los estudios desarrollados sobre osteoporosis, se presta especial atención a las mujeres en edad postmenopáusica precoz, pues es cuando se producen las mayores pérdidas de masa ósea, no existiendo tantos estudios sobre un grupo de población cada día más numeroso y que requiere mayores cuidados, como son los ancianos de más de 70 años.

Los objetivos del presente trabajo son analizar la prevalencia de osteoporosis en una población de ancianos institucionalizados mediante la técnica QUS y contribuir al establecimiento de un punto de corte en unidades de T-score que sea fiable para el diagnóstico de osteoporosis y nos permita avanzar en la prevención de fracturas.

\section{MATERIAL Y MÉTODOS}

\section{DISEÑO}

Se trata de un estudio descriptivo transversal en dos residencias de ancianos, una en el medio rural $(n=120)$ y otra en el urbano $(n=120)$ con una población total de 240 personas. Todos fueron informados de los objetivos del trabajo y accedieron voluntariamente a participar en el estudio tras firmar un consentimiento informado. Después de descartar a los ancianos que por su estado de salud o por la medicación que tomaban pudieran interferir con los resultados, se recogieron datos de 171 ancianos, 111 mujeres (60 de la urbana y 51 de la rural), y 60 varones (26 de la urbana y 34 de la rural).

Se diseñó por parte del equipo de investigación una encuesta que permitía obtener de cada individuo las siguientes variables:

—Datos de filiación: residencia, nombre y apellidos, sexo $\mathrm{y}$ edad.

-Antropométricos: talla $(\mathrm{cm})$, peso $(\mathrm{kg})$ y el cálculo del índice de masa corporal (IMC, $\mathrm{kg} / \mathrm{m}^{2}$ ).

-Ejercicio físico: "bajo" si es menor a 30 minutos diarios; "medio" si está entre 30 y 90 minutos; "alto" si supera los 90 minutos diarios.

-Ultrasonográficos. Se realizó una prueba con un aparato de ultrasonografía cuantitativa de calcáneo (Sahara Clinical Bone Sonometer; Hologic, Inc. USA) siguiendo las instrucciones del fabricante, y calibrado para la población española. Con él se determinaron las siguientes variables: SOS (velocidad del sonido al atravesar el hueso, medido en m/seg); BUA (atenuación del sonido al atravesar el hueso, medido en
$\mathrm{dB} / \mathrm{mgHz}$ ); DMOe (densidad de masa ósea estimada, medido en $\mathrm{g} / \mathrm{cm}^{2}$ ); y el QUI (índice de ultrasonografía cuantitativa, sin unidades); estas dos últimas medidas se calculan a partir de SOS y BUA (7); y la T-score (desviaciones estándar por debajo de la media, comparando al paciente con la población joven de su entorno).

\section{ANÁLISIS ESTADÍSTICO}

El análisis de los resultados se ha realizado con el programa estadístico SPSS versión 12.0. Mediante el test t de Student se hicieron comparaciones de muestras independientes por sexos (varones por un lado y mujeres por otro) entre ambas residencias, tanto para las variables antropométricas como para los parámetros ultrasonográficos. Asimismo se buscaron diferencias entre sexos para el total de la población y por grupos de edad. Para ello se distribuyó a la población en tres grupos: G1: 70-79 años; G2: 80-89 años; G3: 90-99 años.

Para comparar entre varones y mujeres el nivel de actividad física que desarrollaban se aplicó la prueba no paramétrica "U" de Mann-Whitney.

También se ha calculado la prevalencia de osteoporosis aplicando los criterios diagnósticos de la OMS, y los criterios de diferentes autores (8-11):

1. Criterios de la OMS: normal : T-score $\geq-1$; osteopenia: Tscore $<-1$; osteoporosis: T-score $<-2.5$.

2. Criterio de Ayers y cols. : osteoporosis con T-score $<-1$.

3. Criterio de López y cols.: osteoporosis con T-score $<-1.5$.

4. Criterio de Blake y cols.: osteoporosis con T-score $<-1,8$.

\section{RESULTADOS}

Primero se han comparado las variables estudiadas entre ambas residencias, teniendo en cuenta el sexo y no se observaron diferencias estadísticamente significativas en ninguna de las variables. A partir de aquí, se consideró a toda la población en conjunto para el resto del estudio, distribuyéndola por grupos de edad según se ha descrito en el apartado de material y métodos.

\section{DATOS ANTROPOMÉTRICOS}

La comparación entre ambos sexos (Tabla I), considerando la población total, mostró que los varones tienen más estatura, pesan más y la media de edad es menor que la de las mujeres. Sin embargo, la diferencia en el IMC no llega a ser significativa. Al establecer la comparación por grupos de edad se mantuvieron las diferencias en la talla. El peso fue superior en los varones en los grupos G1 y G2. Sin embargo, el IMC fue superior en las mujeres en el grupo G2. Lógicamente desaparecen las diferencias en la edad al distribuir a la población por décadas.

\section{EJERCICIO FÍSICO}

El grado de actividad física que realizan los ancianos, analizado mediante el test no paramétrico ("U" de Mann-Whitney), indica que los varones son más activos que las mujeres, considerando tanto a la población total (significación asintóti- 
TABLA I

CARACTERÍSTICAS ANTROPOMÉTRICAS BÁSICAS DE LA POBLACIÓN OBJETO DE ESTUDIO

\begin{tabular}{|c|c|c|c|c|c|}
\hline Edad & $\operatorname{Sexo}(n)$ & Talla $(\mathrm{cm})$ & Peso $(\mathrm{kg})$ & $I M C\left(k g / m^{2}\right)$ & Edad (años) \\
\hline $\begin{array}{c}70-99 \\
\text { (Total) } \\
p\end{array}$ & $\begin{array}{l}\text { O(111) } \\
\sigma^{\prime}(60)\end{array}$ & $\begin{array}{c}145,9 \pm 6,1 \\
159,3 \pm 6,9 \\
0,000\end{array}$ & $\begin{array}{c}60,05 \pm 10,28 \\
69,25 \pm 10,81 \\
0,000\end{array}$ & $\begin{array}{c}27,08 \pm 4,31 \\
26,03 \pm 3,35 \\
0,107\end{array}$ & $\begin{array}{c}85,7 \pm 5,4 \\
82,6 \pm 6,2 \\
0,001\end{array}$ \\
\hline $\begin{array}{l}70-79 \\
(\mathrm{G} 1) \\
\mathrm{p}\end{array}$ & $\begin{array}{l}\text { O (16) } \\
O^{\prime \prime}(19)\end{array}$ & $\begin{array}{c}147,7 \pm 7,4 \\
159,3 \pm 5,8 \\
0,000\end{array}$ & $\begin{array}{c}61,68 \pm 9,44 \\
70,25 \pm 9,90 \\
0,011\end{array}$ & $\begin{array}{c}27,39 \pm 4,13 \\
26,40 \pm 3,25 \\
0,413\end{array}$ & $\begin{array}{c}77,2 \pm 1,8 \\
76,6 \pm 2,1 \\
0,367\end{array}$ \\
\hline $\begin{array}{l}80-89 \\
(G 2) \\
p\end{array}$ & $\begin{array}{l}\text { }(67) \\
O^{\prime \prime}(33)\end{array}$ & $\begin{array}{c}145,5 \pm 6,3 \\
159,2 \pm 7,3 \\
0,000\end{array}$ & $\begin{array}{c}60,56 \pm 11,61 \\
67,83 \pm 10,93 \\
0,004\end{array}$ & $\begin{array}{c}27,32 \pm 4,54 \\
25,47 \pm 2,99 \\
0,038\end{array}$ & $\begin{array}{c}84,9 \pm 2,6 \\
84,2 \pm 3,0 \\
0,261\end{array}$ \\
\hline $\begin{array}{l}90-99 \\
(G 3) \\
p\end{array}$ & $\begin{array}{l}\text { O (28) } \\
\text { Or (8) }\end{array}$ & $\begin{array}{c}145,6 \pm 4,6 \\
158,9 \pm 9,8 \\
0,011\end{array}$ & $\begin{array}{c}57,49 \pm 6,11 \\
72,55 \pm 14,63 \\
0,053\end{array}$ & $\begin{array}{c}26,20 \pm 3,87 \\
27,53 \pm 5,41 \\
0,500\end{array}$ & $\begin{array}{c}92,6 \pm 2,1 \\
93,3 \pm 3,9 \\
0,554\end{array}$ \\
\hline
\end{tabular}

p: Valor de la p en la prueba de la t de Student. Grado de significación bilateral. Intervalo de confianza del 95\%.

ca bilateral 0.001) como al grupo G2 (significación asintótica bilateral 0,024). Se da la circunstancia que ninguna mujer manifestó un nivel de ejercicio de la categoría alto, mientras que los varones sí lo hicieron.

\section{DATOS ULTRASONOGRÁFICOS}

La comparación de los parámetros ultrasonográficos entre varones y mujeres (Tabla II), tanto de forma global como en los grupos de edad más jóvenes ( $\mathrm{G} 1$ y G2) mostró valores significativamente más bajos en las mujeres para todas las variables. En el grupo de mayor edad (G3) solo se aprecian diferencias significativas en la $\mathrm{T}$-score y en el BUA. No obstante, la mujer presenta también una tendencia a valores inferiores a los del varón en las variables QUI, DMOe y SOS. La figura 1 muestra la evolución de la DMOe en los tres grupos de edad y las diferencias existentes entre varones y mujeres.

En la tabla III se muestra la prevalencia de osteoporosis aplicando diferentes criterios diagnósticos en base a la T-score obtenida mediante la técnica QUS. Bajo cualquiera de los criterios aplicados y en todos los grupos de edad, la prevalencia en las mujeres es superior a la del varón. Cuando hemos aplicado los criterios de la OMS con la técnica QUS, la prevalencia de osteoporosis en la población total se acerca a la establecida mediante DEXA con esos mismos criterios (T-score por debajo de 2,5 desviaciones estándar del valor medio de la población joven en el entorno correspondiente).

TABLA II

RESULTADOS DE LA ULTRASONOGRAFÍA DE CALCÁNEO DISTRIBUIDOS POR SEXOS Y POR GRUPOS DE EDAD

\begin{tabular}{|c|c|c|c|c|c|c|}
\hline Edad & $\operatorname{Sexo}(n)$ & T-score & QUI & DMOe & $B \cup A$ & SOS \\
\hline $\begin{array}{c}\text { 70-99 } \\
\text { (Total) } \\
p\end{array}$ & $\begin{array}{c}\text { O (111) } \\
\sigma^{\prime} \quad(60)\end{array}$ & $\begin{array}{c}-2,08 \pm 0,98 \\
-1,00 \pm 1,28 \\
0,000\end{array}$ & $\begin{array}{c}68,8 \pm 16,3 \\
90,9 \pm 21,8 \\
0,000\end{array}$ & $\begin{array}{c}0,358 \pm 0,103 \\
0,499 \pm 0,138 \\
0,000\end{array}$ & $\begin{array}{c}49,1 \pm 17,2 \\
72,4 \pm 21,0 \\
0,000\end{array}$ & $\begin{array}{c}1511,1 \pm 25,2 \\
1542,2 \pm 34,2 \\
0,000\end{array}$ \\
\hline $\begin{array}{l}70-79 \\
(\mathrm{G} 1) \\
\mathrm{p}\end{array}$ & $\begin{array}{c}\text { O (16) } \\
\text { o (19) }\end{array}$ & $\begin{array}{c}-2,04 \pm 1,04 \\
-0,63 \pm 1,17 \\
, 001\end{array}$ & $\begin{array}{c}70,2 \pm 17,8 \\
97,2 \pm 19,9 \\
0,000\end{array}$ & $\begin{array}{c}0,362 \pm 0,109 \\
0,538 \pm 0,125 \\
0,000\end{array}$ & $\begin{array}{c}52,6 \pm 18,5 \\
78,2 \pm 17,1 \\
, 000\end{array}$ & $\begin{array}{c}1510,0 \pm 25,8 \\
1552,1 \pm 34,1 \\
0,000\end{array}$ \\
\hline $\begin{array}{l}80-89 \\
(G 2) \\
p\end{array}$ & $\begin{array}{l}q(67) \\
\text { or (33) }\end{array}$ & $\begin{array}{c}-2,04 \pm 1,00 \\
-1,18 \pm 1,26 \\
0,000\end{array}$ & $\begin{array}{c}69,3 \pm 16,5 \\
88,1 \pm 21,4 \\
0,000\end{array}$ & $\begin{array}{c}0,362 \pm 0,105 \\
0,481 \pm 0,135 \\
0,000\end{array}$ & $\begin{array}{c}49,0 \pm 16,6 \\
70,3 \pm 21,7 \\
, 000\end{array}$ & $\begin{array}{c}1512,5 \pm 26,6 \\
1537,3 \pm 32,4 \\
0,000\end{array}$ \\
\hline $\begin{array}{l}90-99 \\
(G 3) \\
p\end{array}$ & $\begin{array}{l}\text { O(28) } \\
+(8)\end{array}$ & $\begin{array}{c}-2,20 \pm 0,91 \\
-1,20 \pm 1,71 \\
0,039\end{array}$ & $\begin{array}{c}66,8 \pm 15,1 \\
87,3 \pm 29,3 \\
0,117\end{array}$ & $\begin{array}{c}0,347 \pm 0,096 \\
0,476 \pm 0,185 \\
0,117\end{array}$ & $\begin{array}{c}47,4 \pm 18,2 \\
67,5 \pm 27,6 \\
0,025\end{array}$ & $\begin{array}{c}1508,3 \pm 21,8 \\
1538,2 \pm 44,5 \\
0,130\end{array}$ \\
\hline
\end{tabular}

p: Valor de la p en la prueba de la T de Student. Grado de significación bilateral. Intervalo de confianza del 95\%. 


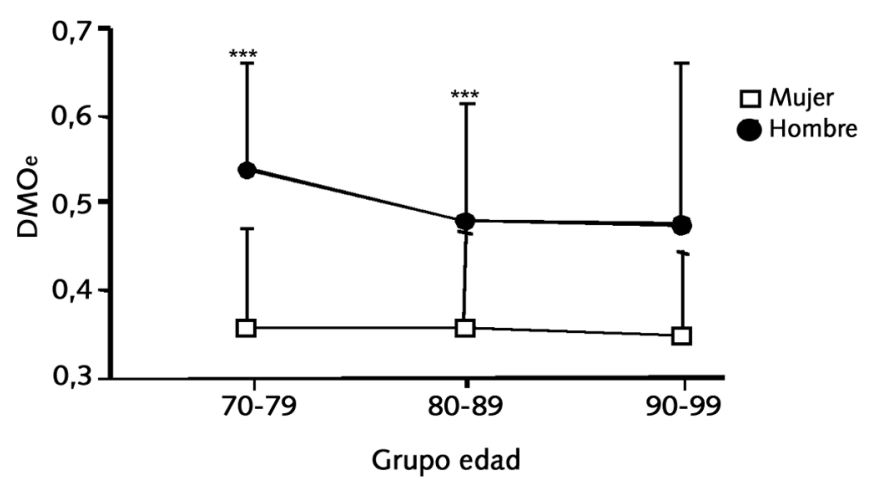

Fig. 1. Densidad de masa ósea estimada.

\section{DISCUSIÓN}

Los resultados referentes a las variables antropométricas comparando varones y mujeres no parecen susceptibles de discusión pues coinciden con numerosos trabajos y es bien conocido que el varón adulto supera a la mujer en talla y en peso. Cabe sin embargo preguntarse porqué existen diferencias en el IMC del grupo 2 (80-89 años). Creemos que puede ser debido a un mayor sedentarismo de las mujeres con relación a los varones. En este sentido, la encuesta sobre actividad física mostraba que las mujeres y los varones del grupo G1 mantienen niveles de actividad física similares, sin embargo muestran diferencias significativas a partir de los 80 años. Parece ser que el varón conserva más tiempo el hábito del paseo, mientras que la mujer se vuelve más sedentaria, afectando a su IMC.

Conforme pasa el tiempo, la población anciana presenta una mayor expectativa de vida, y la incidencia de osteoporosis va a alcanzar cifras muy altas en fechas no muy lejanas, si no se ponen en marcha medidas preventivas eficaces $(1,2,4)$. Aunque, los expertos no recomiendan el cribado general de osteoporosis para toda la población mediante DXA, los USPSTF (U.S. Preventive Services Task Force) sí lo aconsejan en mujeres mayores de 65 años y de entre 60-65 años cuando presentan otros factores de riesgo o fractura previa. Por su parte, la Sociedad Internacional de Densitometría Clínica (ISCD) también propone realizarla en varones mayores de 70 años (12). Un estudio reciente desarrollado por Aragonés y colaboradores concluye que en España "el abordaje de la osteoporosis en atención primaria es deficiente y en parte viene condicionado por la dificultad en el acceso a pruebas diagnósticas y a la falta de sistematización en su cribado" (6). El grupo de población que nos ocupa, tiene además dificultades de acceso a los sistemas de diagnóstico como la DXA (hasta ahora considerada como la técnica más fiable), por circunstancias como la disponibilidad de aparatos en sus lugares de residencia, la movilidad, etc.

Por todo ello nos hemos interesado en las posibilidades que ofrece la técnica QUS. Son numerosos los trabajos que le confieren una capacidad de predicción de fracturas comparable a la DXA (13-15) y, aunque la correlación que existe entre ambas técnicas no es muy alta $(r=0,3-0,8)$ (depende del aparato y la localización de la determinación), ello puede deberse a que no valoran exactamente lo mismo $(10,16,17)$. En nuestra opinión esto no debiera suponer un prejuicio y rechazar de entrada su utilización. A este respecto, diversos autores afirman que la QUS evalúa la microarquitectura ósea y además proporciona un valor estimativo de la densidad de la masa ósea $(5,18,19)$.

En el estudio desarrollado hemos comprobado que la evolución del hueso en varones y en mujeres es diferente, como ya se había observado en otros estudios con la técnica QUS $(7,15)$. El trabajo de Sosa y cols. muestra que el Índice QUI es diferente para varones y mujeres en el grupo de edad de los 70 años y más (15). Sin embargo, no se detalla qué ocurría concretamente en cada década a partir de los 70 años. A pesar de que en nuestro trabajo la población por grupos de edad no es demasiado amplia, coincidiendo con los datos aportados por Sosa y cols., el varón siempre muestra valores ultrasonográficos más altos que la mujer (Tabla II y Fig. 1). Pero además, mientras en las mujeres la DMOe no sufre modificaciones muy llamativas a lo largo de las tres décadas (desciende alre-

TABLA III

PREVALENCIA DE OSTEOPOROSIS EN LA POBLACIÓN ESTUDIADA MEDIANTE QUS EN BASE A DIFERENTES CRITERIOS

\begin{tabular}{|c|c|c|c|c|c|}
\hline & & & US & & DXA (lumbar) \\
\hline & $\begin{array}{c}\text { Ayers } \\
T \text {-score }<-1\end{array}$ & $\begin{array}{c}\text { López } \\
\text { T-score }<-1,5\end{array}$ & $\begin{array}{c}\text { Blake } \\
\text { T-score }<-1,8\end{array}$ & $\begin{array}{c}\text { OMS } \\
T \text {-score }<-2,5\end{array}$ & $\begin{array}{c}\text { OMS } \\
T \text {-score }<-2,5\end{array}$ \\
\hline $\begin{array}{l}\text { Total } \\
\text { Mujer } \\
\text { Varón }\end{array}$ & $\begin{array}{l}81,1 \\
56,7\end{array}$ & $\begin{array}{c}71,2 \\
40\end{array}$ & $\begin{array}{l}60,4 \\
28,3\end{array}$ & $\begin{array}{c}42,3 \\
8,3\end{array}$ & \\
\hline $\begin{array}{l}\text { 70-79 } \\
\text { Mujer } \\
\text { Varón }\end{array}$ & $\begin{array}{l}87,5 \\
36,8\end{array}$ & $\begin{array}{l}75,0 \\
31,6\end{array}$ & $\begin{array}{l}56,3 \\
21,1\end{array}$ & $\begin{array}{c}43,8 \\
5,3\end{array}$ & $\begin{array}{l}40,0 \\
11,3\end{array}$ \\
\hline $\begin{array}{l}\text { 80-89 } \\
\text { Mujer } \\
\text { Varón }\end{array}$ & $\begin{array}{l}76,1 \\
66,7\end{array}$ & $\begin{array}{l}70,1 \\
42,4\end{array}$ & $\begin{array}{l}62,7 \\
30,3\end{array}$ & $\begin{array}{c}41,8 \\
9,1\end{array}$ & \\
\hline $\begin{array}{l}\text { 90-99 } \\
\text { Mujer } \\
\text { Varón }\end{array}$ & $\begin{array}{l}89,3 \\
57,1\end{array}$ & $\begin{array}{l}71,4 \\
57,1\end{array}$ & $\begin{array}{l}57,1 \\
42,9\end{array}$ & $\begin{array}{c}50 \\
28,6\end{array}$ & \\
\hline
\end{tabular}

Datos expresados en porcentaje (\%). 
dedor del 4,2\%), en el varón el descenso alcanza un 11,5\%. El hueso de la mujer parece haber alcanzado los niveles más bajos de masa ósea antes que el varón, probablemente debido a la rápida pérdida postmenopáusica. En el varón, a pesar de no existir diferencias significativas por grupos de edad, se produciría una progresiva tendencia hacia valores más osteoporóticos entre los 70 y los 90 años, descenso que en la mujer se produce previamente debido a la menopausia.

Otra cuestión muy debatida a la hora de aplicar la técnica QUS es el establecimiento de un punto de corte, en términos de T-score, que sirva para: a) establecer el diagnóstico prefractura; b) tenerlo como punto de referencia en el seguimiento de la enfermedad; c) poder pronosticar el riesgo de fractura. Cuando se comenzó a emplear la técnica QUS en el diagnóstico de osteoporosis, se aplicaron los mismos criterios que estableció la OMS para la técnica DXA (20). Sin embargo, existe la duda de si estos criterios son válidos, pues incluso los mismos autores en otro trabajo anterior afirman que dichos criterios minusvaloran el diagnóstico (21). A partir de esta situación, en diferentes estudios se han propuesto puntos de corte distintos para diagnosticar osteoporosis mediante QUS, en un rango de T-score que va desde $(-1)$ hasta $(-1,8)(9-11,15)$. Este diferente nivel de exigencia, provoca inevitablemente que el mismo paciente pueda ser considerado osteoporótico, o no, con la consiguiente repercusión en la prescripción de tratamiento, en su coste económico y en las medidas preventivas. Sosa y cols. (15) toman como criterio una T-score menor de -

\section{Bibliografía}

1. Guía de buena práctica clínica en Geriatría: Osteoporosis. Sociedad Española de Geriatría y Gerontología y Scientific Communication Management, 2004.

2. Grupo Osteoporosis de la semFYC. Osteoporosis. Guía de abordaje. Recomendaciones semFYC. Barcelona: EDIDE, 2000.

3. Díaz Curiel M, García JJ, Carrasco JL, Honorato J, Pérez Cano R, Rapado A, et al. Prevalencia de osteoporosis determinada por densitometría en la población femenina española. Med Clin (Barc) 2001; 116: 86-8.

4. International Osteoporosis Fundation (IOF). Osteoporosis in the European Community: a call to action. An audit of policy development since 1998. IOF. 2001, Brussels.

5. Gluer CC. Quantitative ultrasound Techniques for the assessment of osteoporosis: expert agreement on current status. The International Quantitative Ultrasound Consensus Group. J Bone Miner Res 1997; 12: 1280-8.

6. Aragonés R, Orozco P. y grupo de osteoporosis de la Societat Catalana de Medicina Familiar i Comunitaria. Aten Primaria 2002; 30: 350-6.

7. Sosa M, Saavedra P, Alegre J, Gómez C, González J, Guañabens N, et al. Grupo de investigación en ultrasonidos y metabolismo mineral (GIUMO). Prevalencia de osteoporosis en la población española por ultrasonografía de calcáneo en función del criterio diagnóstico utilizado. Datos del estudio GIUMO. Rev Clin Esp 2003; 203: 329-33.

8. World Health Organization. Assessment of fracture risk and its application to screening for postmenopausal osteoporosis. WHO Genève: WHO Technical Report Series 843, 1994.

9. Ayers M, Prince M, Ahmadi S, Baran DT. Reconciling quantitative ultrasound of the calcaneus with X-ray-based measurements of the central skeleton. J Bone Miner Res 2000; 15: 1850-5.

10. López-Rodríguez F, Mezquita-Raya P, Luna JD, Escobar-Jiménez F, Muñoz-Torres M. Performance of quantitative ultrasound in the discrimination of prevalent osteoporotic fractures in a bone metabolic unit. Bone 2003; 32: 571-8

11. Blake DM, Knapp K, Fogelman I. Should equivalent T-score be based on absolute fracture risk? Osteoporos Int 2001; 12 (Supl. 2): S8-9.

12. U.S. Preventive Services Task Force. Screening for osteoporosis in postmenopausal women: recommendations and rationale. Ann Intern Med
1.8 pues de esta forma, en su estudio, la prevalencia de osteoporosis mediante QUS se acerca más a las cifras obtenidas con DXA $(3,22)$ pero concluyen que es necesario un consenso para establecer cuál es el punto de corte adecuado que sirva para diagnosticar la osteoporosis mediante QUS.

En nuestro estudio, cualquier criterio aplicado, diferente al de la OMS, ofrecía valores de prevalencia muy altos, comparados con los de referencia obtenidos con DXA $(3,22)$, como se aprecia en la tabla III. Sin embargo, al aplicar los criterios de la OMS, obtuvimos una prevalencia, global y por edades que se ajusta más a los valores de referencia.

En conclusión, creemos que la técnica QUS puede tener una gran utilidad a la hora de valorar cómo evoluciona la masa ósea con la edad y por lo tanto en el diagnóstico y seguimiento de la osteoporosis. Por otra parte, y a la luz de los resultados aportados, en nuestra población anciana, los criterios de la OMS para la técnica DXA, son los más adecuados para el empleo de la técnica QUS.

\section{AGRADECIMIENTOS}

Expresamos nuestro agradecimiento al Profesor Fernando J. Chueca Moncayo, del Dpto. de Filología Inglesa de la Facultad de Traducción e Interpretación de Soria (Universidad de Valladolid), por su asesoramiento en la elaboración del texto en lengua Inglesa.
2002; 137: 526-8.

13. Huopio J, Kröger H, Honkanen R, Jurvelin J, Saarikoski S, Alhava E. Calcaneal ultrasound predicts early postmenopausal fractures as well as axial BMD. A prospective study of 422 women. Osteoporos Int 2004; 15: 190-5.

14. Miller PD, Siris ES, Barrett-Connor E, Faulkner KG, Wehren LE, Abbott TA, et al. Prediction of fracture risk in postmenopausal white women with peripheral bone densitometry: evidence from the National Osteoporosis Risk Assessment. J Bone Miner Res 2002; 17: 222230.

15. Sosa M, Saavedra P, Muñoz-Torres M, Alegre J, Gómez C, GonzálezMacías J, et al and the GIUMO Study Group. Quantitative ultrasound calcaneus measurements: normative data and precision in the Spanish population. Osteoporos Int 2002; 13: 487-92.

16. Faulkner K, McClung M, Coleman L, Kingston-Sadahl E. Quantitative ultrasound of the heel: correlation with densitometric measurements at different skeletal sites. Osteoporos Int 1994; 4: 42-7.

17. Espallargues M, Estrada MD, Sampietro-Colom L, Sola M, del Rio L, Granados A. Guía para la indicación de la densitometría ósea en la valoración del riesgo de fractura.: Agència d'Avaluació de Tecnologia Mèdica. Servei Català de la Salut. Departament de Sanitat i Seguretat Social. Generalitat de Catalunya; Barcelona, 1999. (a)

18. Kaufman JJ, Einhorn TA. Perspective: ultrasound assessment of bone. Osteoporos Int 1993; 8: 517-25.

19. Gluer CC, Barkmann R. Quantitative ultrasound: use in the detection of fractures and in the assessment of bone composition. Curr Osteoporos Rep 2003; 1: 98-104.

20. Frost ML, Blake GM, Folgeman I. Quantitative ultrasound and bone mineral density are equally strongly associated with risk factors for osteoporosis. J Bone Miner Res 2001; 16: 406-16.

21. Frost ML, Blake GM, Folgeman I. Can the WHO criteria for diagnosing osteoporosis be applied to calcaneal quantitative ultrasound? Osteoporos Int 2000; 11: 321-30.

22. Díaz M, Turbí C, Rapado A, García JJ. Prevalencia de osteopenia y osteoporosis densitométrica en la población masculina española. Rev Esp Enf Metab Óseas 1997; 6: 129-32. 